\title{
两亲聚肽/螺烯的电荷复合自组装行为研究
}

\author{
王婧琳 ${ }^{a}$ 沈程硕 $b$ 唐颂超*,a 姚 远*,a \\ ( ${ }^{a}$ 华东理工大学材料科学与工程学院＼cjkstart高分子材料与工程教研室上海 200237) \\ $\left({ }^{b}\right.$ 上海交通大学化学化工学院 上海 200240)
}

\begin{abstract}
摘要 研究了包含聚肽嵌段的两亲共聚物聚乙二醇 $-b$ - 聚( $(L$-谷氨酸)(PEG- $b$-PGlu) 与 4 -甲基-4-氮杂 $[4]$ 螺烯鎓离子 $(\mathrm{Me}[4] \mathrm{H})$ 的电荷复合自组装行为, 讨论了聚肽链段长度、谷氨酸单元与螺烯物质的量比、 $\mathrm{pH}$ 值等因素对组装体形貌的 影响. 研究发现, 当刚-柔型嵌段聚合物与刚性、大尺寸并具有诱导作用的小分子共同组装时, 遵循与柔性大分子电荷 复合组装完全不同的规律. PEG- $b$-PGlu/Me[4]H 的电荷复合组装体形貌由 $\pi-\pi$ 堆叠作用、两亲嵌段聚肽的亲疏水比、PGlu 链段的刚硬程度共同决定, 这些作用在组装过程中相互制约, 当 $\pi-\pi$ 堆叠作用占主导地位时, 得到以指针状和条带状等 平面结构为主的组装形貌.
\end{abstract}

关键词＼cjkstart电荷复合自组装; 两亲嵌段共聚物; 螺烯

\section{Study of Charge-Conjugated Self-Assembly Behavior of Amphiphilic Block Copolypeptides/Helicene}

\author{
Wang, Jinglin $^{a} \quad$ Shen, Chengshuo ${ }^{a} \quad$ Tang, Songchao*,a Yao, Yuan ${ }^{*, a}$ \\ ( ${ }^{a}$ Teaching and Research Department of Polymer Materials and Engineering, School of Materials Science and Engineering, \\ East China University of Science and Technology, Shanghai 200237) \\ ( ${ }^{b}$ School of Chemistry and Chemical Engineering, Shanghai Jiao Tong University, Shanghai 200240)
}

\begin{abstract}
Amphiphilic block copolypeptides of polyethylene glycol- $b$-poly ( $L$-glutamic acid) (PEG- $b$-PGlu) were synthesized to self-assemble with 4-methyl-4-aza[4]helicene onium ion $(\mathrm{Me}[4] \mathrm{H})$ in water through charge-conjugation. The morphology of the assemblies was studied by varying PGlu block length, the molar ratio of Glu unit/Me[4]H and pH value. It was found that when rigid-flexible block copolymers were assembled together with small molecules which had rigid, large size and inductive effect, they would follow a completely different law from the charge-conjugated assembly of flexible macromolecules. The self-assemblies were controlled by the $\pi-\pi$ stacking of helicene, together with the volume fraction of the hydrophobic parts and the rigidity of PGlu segments. These effects restricted each other in the assembly process. When $\pi-\pi$ stacking was dominant, assembly morphologies with pointer-like and strip-like planar structures were obtained.

Keywords charge-conjugated self-assembly; amphiphilic block copolymer; helicene
\end{abstract}

电荷复合自组装是广受关注的一类自组装形

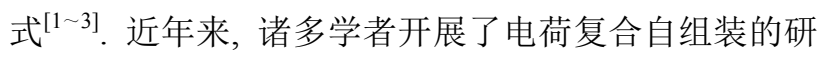
究, 使得电荷复合自组装得以被应用于光伏 ${ }^{[4,5]}$ 、表面 处理 ${ }^{[6-8]}$ 、光电器件 ${ }^{[9-11]}$ 、膜技术 ${ }^{[12 \sim 15]}$ 、传感器 ${ }^{[16 ~ 18] 、 ~}$

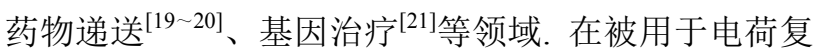
合自组装的高分子中, 聚肽由于可在不同条件下表现 出不同的构象和组装行为, 给予了电荷复合自组装更 多的可能性而受到广泛关注 ${ }^{[22 ~ 30]}$. 利用聚肽与小分子 和聚电解质分子电荷复合自组装, 可以得到球状胶束、
棒状胶束、囊泡、螺旋飘带等多种组装形貌. 当聚肽嵌 段展现出手性二级结构时, 通常显示为刚硬的螺旋结 构, 所以与聚肽电荷复合的物质一般为自由度非常高 的小分子或柔软链段的大分子，这些化合物不会对组 装体的形貌产生引导作用. 然而, 在聚合物的电荷复 合自组装中，如果发生相互作用的正负电荷部分均为 刚硬的构象，它们将遵循何种组装规律，目前还没有 被深入探讨. 这也是自组装领域令人感兴趣的课题之 一.

\footnotetext{
* Corresponding authors. E-mail: schtang@ecust.edu.cn; yaoyuan@ecust.edu.cn Received April 15, 2019; revised May 7, 2019; published online May 21, 2019. Project supported by the National Natural Science Foundation of China (No. 51573088). 国家自然科学基金(No. 51573088)资助项目.
} 
我们引入本身对组装具有引导作用、又可与聚肽发 生电荷相互作用, 并且具备较高自由度的刚硬大尺寸小 分子 4-甲基-4-氮杂 [4]螺烯鎓离子 $(\mathrm{Me}[4] \mathrm{H})$ 与两亲嵌段 聚肽聚乙二醇- $b$-聚 ( $L$-谷氨酸) (PEG- $b$-PGlu) 进行电荷复 合自组装. 螺烯是一类具有独特的固有手性和光学特性 的芳香分子 ${ }^{[31]}, \mathrm{Me}[4] \mathrm{H}$ 分子具有刚硬的近平面构象, 可 以通过 $\pi-\pi$ 堆叠对自组装产生诱导作用, 也能在组装过 程中随大分子链运动. PEG- $b$-PGlu 则通过端氨基聚乙二 醇 $\left(\mathrm{mPEG}-\mathrm{NH}_{2}\right)$ 引发 $L$-谷氨酸-芳基- $N$ - 羧基内酸䣶 (BLG-NCA)开环聚合, 然后脱去保护基团得到(Scheme $1)^{[32 \sim 34]}$. 在水溶液中, 低 $\mathrm{pH}$ 值和羧基阴离子被中和时, 聚 ( $L$ - 谷氨酸)(PGlu) 链段为刚性的 $\alpha$-螺旋构象, PEG- $b$-PGlu 是刚-柔构象的两亲性聚合物; 高 $\mathrm{pH}$ 值时, 由于羧基电离度增加, 电荷排斥作用导致 $\alpha$-螺旋解体, 两嵌段均表现为柔性的无规线团构象. 通过调节 PGlu 嵌段长度、 $\mathrm{Me}[4] \mathrm{H}$ 加入量以及 $\mathrm{pH}$ 值, 使 $\mathrm{PGlu}$ 与 $\mathrm{Me}[4] \mathrm{H}$ 之间相互作用力、电荷复合部分的疏水性、亲疏水部分 体积分数以及 PGlu 嵌段的构象等因素发生变化, 研究 在 $\pi-\pi$ 堆叠诱导下的刚-柔到柔-柔构象可调的两亲嵌段 聚合物的组装行为, 总结其规律. 本研究不但填补了电 荷复合自组装领域的研究空白, 而且为两亲聚肽嵌段聚 合物载体通过电荷复合作用搭载刚硬构象的功能分子 进行药物递送、制备功能化组装体等应用提供了很有价 值的参考.<smiles>NC(CCC(=O)OCc1ccccc1)C(=O)OCCC(N)C(=O)OCc1ccccc1</smiles>

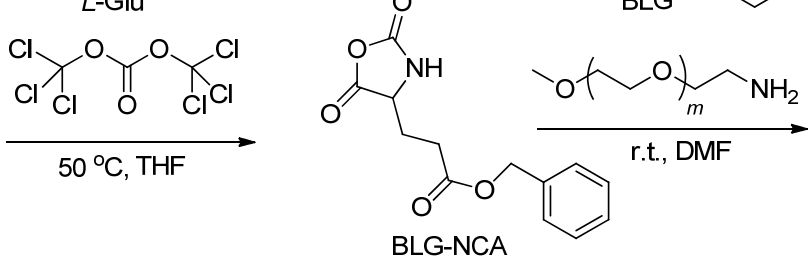<smiles>CCNC(CCC(=O)OCc1ccccc1)C(=O)NCCCOCCOCCOCC</smiles>

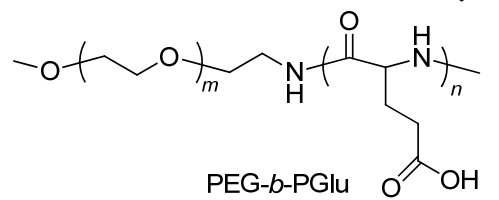

图式 1 PEG- $b$-PGlu 的合成路线 Scheme 1 Synthetic route of PEG- $b$-Pglu

\section{1 结果与讨论}

\subsection{PEG- $b-P G l u$ 和 $\mathrm{Me}[4] \mathrm{H}$ 的表征}

本实验中采用的 $\mathrm{mPEG}-\mathrm{NH}_{2}$ 相对分子量均为 $2.00 \times 10^{3}$, 嵌段数为 45 , 合成的聚乙二醇 $-b$-聚 $(L$-谷氨 酸芐酯)(PEG- $b-\mathrm{PBLG})$ 参数如表 1 中所示, $n$ 表示聚 $(L-$ 谷氨酸芐酯)(PBLG)的嵌段重复单元数. 基于凝胶渗透 色谱(GPC)结果以及每个结构单元的相对分子质量, 计 算出各嵌段共聚物中 PBLG 的实际嵌段数如表 1. 将按 照投料比计算得到的嵌段数结果与 GPC 结果得到的嵌 段数进行对照, 数值相近, 同时, 分子量分布(PDI)均低 于 1.20 , 说明反应按照预期进行 ${ }^{[32 ~ 34]}$. 由于脱保护后难 以准确测得嵌段聚合物分子量, 因此实际 PGlu 嵌段数 以对应 PEG- $b$-PBLG 计算出的嵌段数为准. PEG- $b-$ PBLG 脱保护后得到的 PEG- $b$-PGlu 的核磁共振氢谱 $\left({ }^{1} \mathrm{H}\right.$ $\mathrm{NMR}$ ( $\mathrm{D}_{2} \mathrm{O}, 400 \mathrm{MHz}$ )结果为 $\delta: 4.00 \sim 4.30$ ( $\alpha$-次甲基), $3.30 \sim 3.75$ (聚乙二醇 $(\mathrm{PEG}$ )亚甲基), $2.00 \sim 2.40$ (与羧基 相连的亚甲基), $1.70 \sim 2.00$ (与 $\alpha$-碳相连的亚甲基), 7.30 左右没有芳环的质子峰出现, 表明聚合物脱保护完全.

\subsection{PGlu 嵌段长度对组装体形貌的影响}

将疏水嵌段长度不同的PEG- $b$-PGlu分别与 $\mathrm{Me}[4] \mathrm{H}$ 在水中进行电荷复合自组装, 其中 PEG- $b$-PGlu 与 $\mathrm{Me}[4] \mathrm{H}$ 之间的羧基与鎓离子之比为 $1: 0.5$, 溶液的 $\mathrm{pH}$ 值为 6.0 , 探究疏水链段长度对组装体形貌的影响, 透射 电子显微镜(TEM)照片如图1所示.

由图 $1 \mathrm{~A}$ 可见, $\mathrm{PEG}_{45}-b-\mathrm{PGlu}_{10}$ 与 $\mathrm{Me}[4] \mathrm{H}$ 组装成了长 约 $20 \mu \mathrm{m}$, 宽约 $1 \mu \mathrm{m}$ 的长指针状组装体. 随着PGlu嵌段 数逐渐增加, 加入的 $\mathrm{Me}[4] \mathrm{H}$ 也同比例增加, 组装体中疏 水部分的体积分数逐渐增大, 此时组装体系的形貌变化 规律并不遵循传统的大分子自组装规律，而是得到尺寸 逐渐减小的片状结构. 由图 $1 \mathrm{~B}$ 可见, $\mathrm{PEG}_{45}-b-\mathrm{PGlu}_{24}$ 与 $\mathrm{Me}[4] \mathrm{H}$ 组装成了长度在 $15 \mu \mathrm{m}$ 左右, 最宽约 $1.0 \mu \mathrm{m}$ 的长 指针状组装体. 当PGlu的嵌段数达到 36 时, 组装体变化 为较为均匀的长约 $6.0 \mu \mathrm{m}$, 宽约为 $0.3 \mu \mathrm{m}$ 的条带状组装 体(图1C), 当疏水嵌段数达到 50 时, 组装体长度缩小到 约 $2.5 \mu \mathrm{m}$, 宽度变化不大 (图 1D). $\mathrm{PEG}_{45}-b-\mathrm{PGlu}_{71}$ 与 $\mathrm{Me}[4] \mathrm{H}$ 进行电荷复合形成的短条状组装体长度不超过 $0.5 \mu \mathrm{m}$, 宽约为 $0.1 \mu \mathrm{m}$ (图1E).

由于 PGlu 链段在 $\mathrm{pH}$ 值为 6.0 时并未完全电离，按 照其完全电离的比例 $1 / 2$ 加入反离子 $\mathrm{Me}[4] \mathrm{H}$ 后, PGlu 链段与 $\mathrm{Me}[4] \mathrm{H}$ 发生电荷中和, 同时 $\mathrm{Me}[4] \mathrm{H}$ 的正电荷也 被中和，因此出现的 $\pi-\pi$ 堆叠作用使 $\mathrm{Me}[4] \mathrm{H}$ 排列为片 层状的有序结构 ${ }^{[35 \sim 37]}$ ，作为疏水的内核. PGlu 受到相反 电荷的吸引，也按照 $\mathrm{Me}[4] \mathrm{H}$ 堆叠的方向排列，作为疏 水的外层. 由于嵌段聚合物 $\mathrm{PEG}_{45}-b-\mathrm{PGlu}_{10}$ 亲水链所占 
表 $1 \quad \mathrm{PEG}_{45}-b-\mathrm{PBLG}_{n}$ 的参数

Table 1 Parameters of $\mathrm{PEG}_{45}-b-\mathrm{PBLG}_{n}$

\begin{tabular}{cccccc}
\hline Copolymer & $M_{n(\exp )}{ }^{a}\left(\times 10^{4}\right)$ & $M_{n(\mathrm{obt})}{ }^{b}\left(\times 10^{4}\right)$ & $\mathrm{DP}_{\text {exp,PBLG }}{ }^{c}$ & $\mathrm{DP}_{\text {obt,PBLG }}{ }^{d}$ & PDI \\
\hline $\mathrm{PEG}_{45}-b-\mathrm{PBLG}_{10}$ & 0.38 & 0.40 & 10 & 20 & 1.13 \\
$\mathrm{PEG}_{45}-b-\mathrm{PBLG}_{24}$ & 0.58 & 0.66 & 30 & 24 & 1.18 \\
$\mathrm{PEG}_{45}-b-\mathrm{PBLG}_{36}$ & 0.83 & 0.89 & 50 & 50 & 1.19 \\
$\mathrm{PEG}_{45}-b-\mathrm{PBLG}_{50}$ & 1.10 & 1.17 & 70 & 71 & 1.06 \\
$\mathrm{PEG}_{45}-b-\mathrm{PBLG}_{71}$ & 1.44 & 1.57 & 50 & 1.08 \\
\hline
\end{tabular}

${ }^{a} M_{n(\mathrm{exp})}$ 表示根据投料比计算出的平均分子量 ${ }^{b} M_{n(\mathrm{obt})}$ 表示 $\mathrm{GPC}$ 测出的相对分子质量 ${ }^{c} \mathrm{DP}_{\mathrm{exp}, \mathrm{PBLG}}$ 表示根据投料比计算出的 $\mathrm{PBLG}$ 嵌段数; ${ }^{d} \mathrm{DP}$ obt,PBLG 表示 根据 GPC 结果计算出的 PBLG 嵌段数.
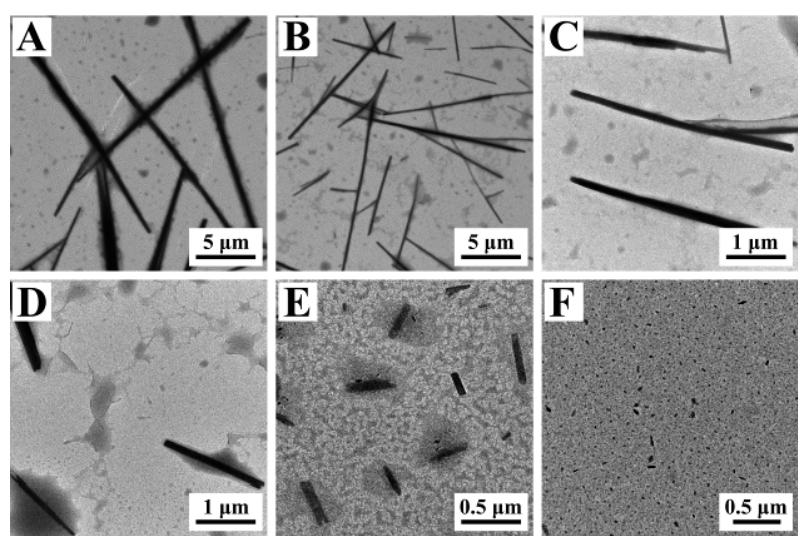

图 1 不同疏水嵌段长度的 PEG- $b$-PGlu 与 $\mathrm{Me}[4] \mathrm{H}$ 在水中电 荷复合自组装的 $\mathrm{TEM}$ 照片 $(\mathrm{pH}=6.0$, 羧基与鎓离子之比为 $1: 0.5)$

Figure 1 TEM images of the self-assembly of PEG- $b$-PGlu with various hydrophobic block lengths charge-conjugated $\mathrm{Me}[4] \mathrm{H}$ in water $(\mathrm{pH}=6.0$, carboxyl group and onium ion ratio is $1: 0.5)$

(A) $\mathrm{PEG}_{45}-b$ - $\mathrm{PGlu}_{10}$; (B) $\mathrm{PEG}_{45}-b-\mathrm{PGlu}_{24} ;$ (C) $\mathrm{PEG}_{45}-b-\mathrm{PGlu}_{36}$; (D) $\mathrm{PEG}_{45}-b$ - $\mathrm{PGlu}_{50}$; (E) $\mathrm{PEG}_{45}-b$ - $\mathrm{PGlu}_{71}$; (F) Me[4]H as a comparison

体积分数相对较多, 可以 “支持” 相对较大的组装体, 因 此在此条件下, $P G_{45}-b-P G u_{10}$ 所得指针形组装体具有 最大的尺度. 当疏水链段体积分数逐渐增加时, 一方面 由于 $\pi-\pi$ 堆叠作用依然存在, 所得组装体的形貌并非按 照柔性两亲大分子自组装的变化规律变化; 另一方面, 组装体尺寸也逐渐变小, 以维持组装体在溶液中的稳 定, 形貌也出现了长指针状到条带状的变化. 由 $\zeta$-电位
测量结果可知, 组装体外部均带有少量的负电荷(表 2), 进一步证明了所有电荷复合组装体疏水部分的片层结 构都是 $\mathrm{Me}[4] \mathrm{H}$ 在内部, PGlu 在外部的夹心状结构(图 2). 此外, 如图 $1 \mathrm{~F}$ 所示, 纯 $\mathrm{Me}[4] \mathrm{H}$ 鎓离子在水中仅能组装 为很小的胶束粒子, 这是由于鎓离子所带正电荷的排斥 作用使之无法 $\pi-\pi$ 堆叠，只能依靠疏水力作用形成小胶 束; 而在 $\mathrm{pH}=6.0$ 时, PEG- $b$-PGlu 的 PGlu 嵌段亲水性已 经变得较强, 也无法得到稳定的组装体. 因此, 长指针 状和条带状组装体是 PEG- $b$-PGlu 与 Me[4]H 电荷复合 自组装形成的.

表 2 不同疏水嵌段长度的 PEG- $b-\mathrm{PGlu}$ 与 $\mathrm{Me}[4] \mathrm{H}$ 在水中电 荷复合自组装的 $\zeta$-电位值 $(\mathrm{pH}=6.0$, 羧基与鎓离子之比为 1 : $0.5)$

Table $2 \zeta$-Potential values of self-assembly of PEG- $b$-PGlu with various hydrophobic block lengths charge-conjugated $\mathrm{Me}[4] \mathrm{H}$ in water $(\mathrm{pH}=6.0$, carboxyl group and onium ion ratio is $1: 0.5)$

\begin{tabular}{lc}
\hline$n^{a}$ & $\zeta$-Potential $/ \mathrm{mV}$ \\
\hline 10 & -5.74 \\
24 & -7.19 \\
36 & -2.45 \\
50 & -1.56 \\
71 & -1.03 \\
\hline
\end{tabular}

${ }^{a} n$ 表示 PGlu 的嵌段数.

\section{$1.3 \mathrm{Glu} / \mathrm{Me}[4] \mathrm{H}$ 物质的量比对组装体形貌的影响}

选择 $\mathrm{PEG}_{45}-b-\mathrm{PGlu}_{10}$ 与 $\mathrm{Me}[4] \mathrm{H}$ 在水中进行电荷复
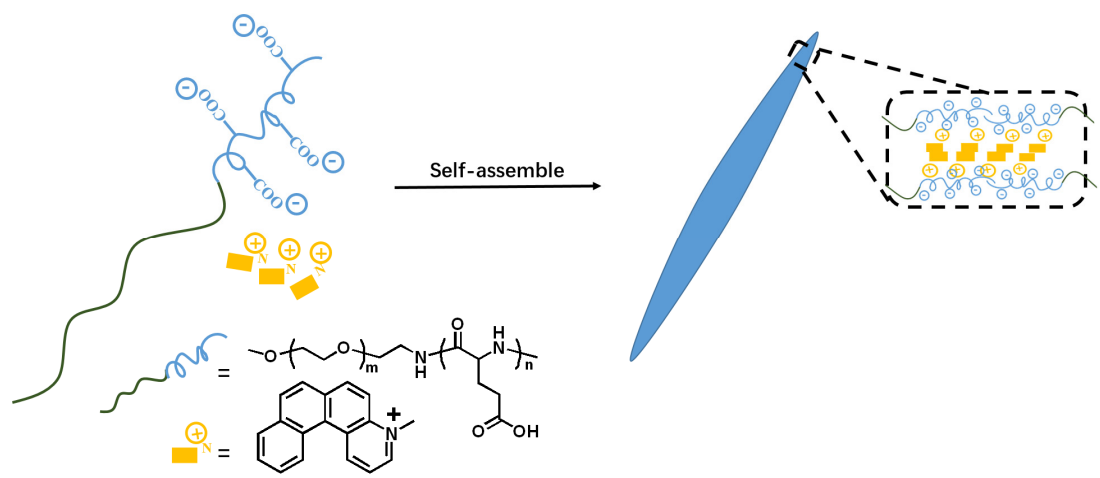

图 2 PEG- $b$-PGlu 与 $\mathrm{Me}[4] \mathrm{H}$ 电荷复合自组装为长指针状的组装体示意图

Figure 2 Charge-conjugated self-assembly of PEG- $b$-PGlu and Me[4]H into long pointer-like morphology 
$0.5 、 1 ： 0.7 、 1 ： 1.0$, 调节溶液的 $\mathrm{pH}$ 值至 6.0 , 以 $\mathrm{TEM}$ 合自组装. 改变 $\mathrm{Me}[4] \mathrm{H}$ 的加入比例, 即 PEG- $b$-PGlu 与 $\mathrm{Me}[4] \mathrm{H}$ 的羧基与鎓离子之比分别为 $1: 0.1 、 1: 0.2 、 1$ : 观察 $\mathrm{Glu} / \mathrm{Me}[4] \mathrm{H}$ 物质的量比变化对组装体的形貌的影 响, 结果如图 3 所示.
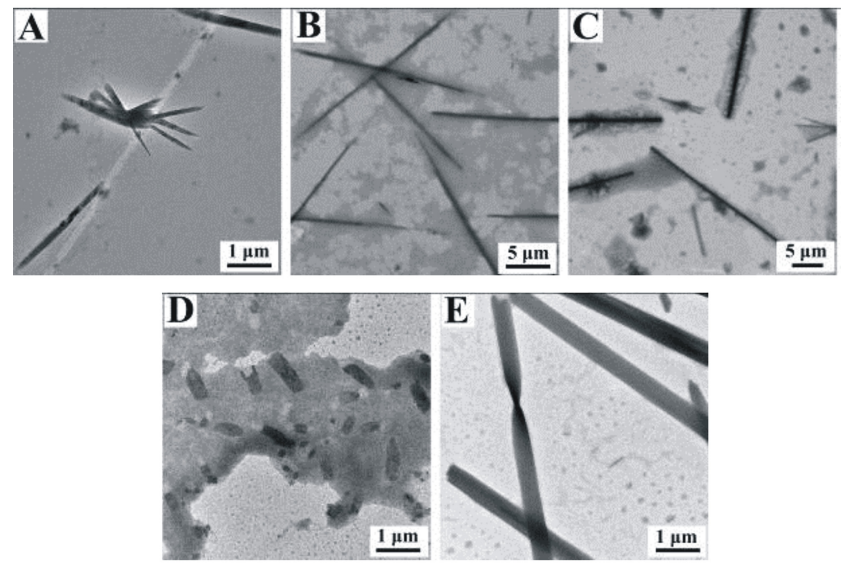

图 $3 \mathrm{PEG}_{45}-b-\mathrm{PGlu}_{10}$ 与 $\mathrm{Me}[4] \mathrm{H}$ 的不同物质的量比下在水中 电荷复合自组装的 TEM 照片 $(\mathrm{pH}=6.0)$

Figure 3 TEM images of the self-assembly of $\mathrm{PEG}_{45}-b$ - $\mathrm{PGlu}_{10}$ charge-conjugated $\mathrm{Me}[4] \mathrm{H}$ with different molar ratios in water $(\mathrm{pH}=6.0)$

(A) $1: 0.1 ;$ (B) $1: 0.2 ;$ (C) $1: 0.5 ;$ (D) $1: 0.7 ;$ (E) $1: 1.0$

当 $\mathrm{PEG}_{45}-b-\mathrm{PGlu}_{10}$ 与 $\mathrm{Me}[4] \mathrm{H}$ 之间羧基与鎓离子之 比为 $1: 0.1$ 时, 得到长度为 $2 \sim 4 \mu \mathrm{m}$, 最宽处为 $0.2 \mu \mathrm{m}$ 左右的指针状组装体(图 3A), 此时, PGlu 链段能够保持 部分 $\alpha$-螺旋构象(图 4). 在自组装过程中, 尽管螺烯的 $\pi-\pi$ 堆叠作用和 PGlu 链段 $\alpha$-螺旋构象的定位作用构成了 竞争关系, 但前者仍起主导作用. 可用于堆叠成疏水内 层的 $\mathrm{Me}[4] \mathrm{H}$ 分子则较少, 因此得到短指针状组装体. 随着 $\mathrm{Me}[4] \mathrm{H}$ 加入量的增加, $\mathrm{Me}[4] \mathrm{H}$ 分子间 $\pi-\pi$ 堆叠的

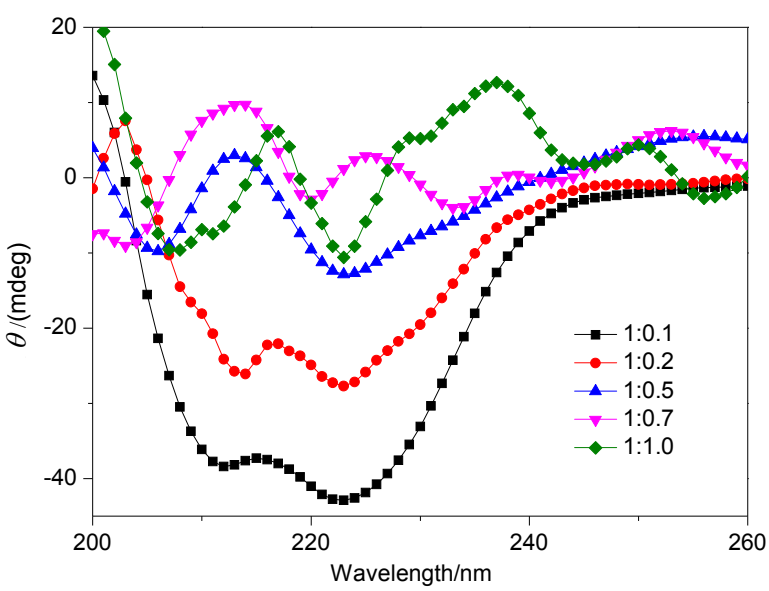

图 $4 \mathrm{PEG}_{45}-b-\mathrm{PGlu}_{10}$ 与 $\mathrm{Me}[4] \mathrm{H}$ 的不同物质的量比下在水中 电荷复合自组装的圆二色谱图 $(\mathrm{pH}=6.0)$

Figure $4 \mathrm{CD}$ spectra of self-assembly of $\mathrm{PEG}_{45}-b$ - $\mathrm{PGlu}_{10}$ charge-conjugated $\mathrm{Me}[4] \mathrm{H}$ with different molar ratios in water $(\mathrm{pH}=6.0)$
主导作用逐渐加强，也使得 PGlu 链段的 $\alpha$-螺旋逐渐被 破坏(图 4). 因此，当羧基与鎓离子之比为 $1: 0.2$ 时，得 到长度达到 $15 \mu \mathrm{m}$ 左右, 最宽处为 $0.6 \sim 0.7 \mu \mathrm{m}$ 的长指 针状组装体(图 3B); 而当羧基与鎓离子之比为 $1: 0.5$ 时，组装体的尺寸可以增大到 $20 \mu \mathrm{m}$ 以上(图 3C).

但是，当羧基与鎓离子之比达到 $1: 0.7$ 时, TEM 只 能观察到很短的条带状组装体, 其长度不到 $1 \mu \mathrm{m}$, 宽度 约为 $0.5 \mu \mathrm{m}$ (图 3D). 当羧基与鎓离子之比达到 $1: 1.0$ 时，又生成了新的条带状组装体，该组装体的宽度与电 荷比 $1: 0.7$ 时得到的组装体宽度几乎一致, 均为 $0.5 \mu \mathrm{m}$ 左右, 但长度可达数十微米(图 3E). 这是因为在羧基与 鎓离子之比达到 $1: 1.0$ 时，可用于构成疏水内层的螺烯 更多，它们在 $\pi-\pi$ 堆叠作用下有序排列，并与对应的 PGlu $_{10}$ 链段共同组装, 形成层状的条带组装结构. 这种 条带状组装体中间部分几乎全部由疏水的螺烯 $\pi-\pi$ 堆叠 构成, 因此其宽度和厚度非常均匀. 而电荷比 $1: 0.7$ 时 得到的组装产物则处于指针状组装体向条带状组装体 转化的过渡区. 此外, 当羧基与鎓离子之比为 $1: 0.5$ 以 上时，已很难观察到 $\alpha$-螺旋构象的圆二色谱 $(\mathrm{CD})$ 信号 (图 4), 这说明 $\mathrm{Me}[4] \mathrm{H}$ 的加入对螺旋的破坏作用很大, 也进一步证明了大尺寸的指针状组装体和条带状组装 体的形成主要是依赖 $\pi-\pi$ 堆叠和更高的 $\mathrm{Me}[4] \mathrm{H}$ 体积分 数.

表 3 显示, 随着 $\mathrm{Me}[4] \mathrm{H}$ 的加入，组装体表面的负电 荷逐步减少，但即使当羧基与鎓离子之比为 $1: 1.0$, 理 论上正电荷数已经过量时(羧基在 $\mathrm{pH}=6.0$ 时不能完全 电离), 组装体的表面仍然覆盖着负电荷. 这表明, 无论 是指针状组装体还是条带状组装体，其外表面均覆盖着 亲水的 PEG 大分子链. 在亲水的 PEG 大分子链的 “拉 拽” 作用和疏水的 $\mathrm{Me}[4] \mathrm{H}$ 本体的排斥下, PGlu 嵌段倾 向于在内层的 $\mathrm{Me}[4] \mathrm{H}$ 表面富集, 形成夹心状的疏水层 结构, 因此 $\zeta$-电位测试结果均为负值, 这一结果进一步 揭示了两种组装体的内部结构的类似性.

表 $3 \mathrm{PEG}_{45}-b-\mathrm{PGlu}_{10}$ 与 $\mathrm{Me}[4] \mathrm{H}$ 的不同物质的量比下在水中 电荷复合自组装的 $\zeta$-电位值 $(\mathrm{pH}=6.0)$

Table $3 \zeta$-Potential values of self-assembly of $\mathrm{PEG}_{45}-b$-PGlu 10 charge-conjugated $\mathrm{Me}[4] \mathrm{H}$ with different molar ratios in water $(\mathrm{pH}=6.0)$

\begin{tabular}{cc}
\hline Glu $: \mathrm{Me}[4] \mathrm{H}$ & $\zeta$-Potential $/ \mathrm{mV}$ \\
\hline $1: 0.1$ & -12.31 \\
$1: 0.2$ & -9.85 \\
$1: 0.5$ & -5.74 \\
$1: 0.7$ & -4.94 \\
$1: 1.0$ & -.72 \\
\hline
\end{tabular}

此外, 我们还选择了 PGlu链更长的 $\mathrm{PEG}_{45}-b-\mathrm{PGlu}_{24}$, 同样与 $\mathrm{Me}[4] \mathrm{H}$ 在水中进行电荷复合自组装. 改变 
$\mathrm{Me}[4] \mathrm{H}$ 的加入比例，即羧基与鎓离子之比分别为 1 : $0.1 、 1: 0.2 、 1: 0.5 、 1: 0.7 、 1: 1.0$, 溶液的 $\mathrm{pH}=6.0$ 时, 进一步研究 $\mathrm{Glu} / \mathrm{Me}[4] \mathrm{H}$ 物质的量比对组装体形貌的 影响.

由 TEM 观察结果可知, $\mathrm{PEG}_{45}-b-\mathrm{PGlu}_{24}$ 在 $\mathrm{Me}[4] \mathrm{H}$ 含量逐渐增加时, 其组装行为与 PGlu 链较短的 $\mathrm{PEG}_{45}-b$ $\mathrm{PGlu}_{10}$ 有很大不同. 当羧基与鎓离子之比为 $1: 0.1 、 1$ : 0.2 时, 溶液中很难观察到规整的组装体(图 5A), 或只 能观察到油滴状物质(图 5B). 直到羧基与鎓离子之比达 到 $1: 0.5$ 时, 体系内才出现长度 $5 \sim 20 \mu \mathrm{m}$, 最宽处为 $0.3 \mu \mathrm{m}$ 左右的长指针状组装体 (图 $5 \mathrm{C}$ ). 当羧基与鎓离子 之比达到 $1: 0.7$ 时, 组装体的宽度略有增加(图 5D). 但 当羧基与鎓离子之比达到 $1: 1.0$ 时, 仅能得到尺寸为 $40 \sim 50 \mathrm{~nm}$ 的胶束(图 $5 \mathrm{E}$ ).

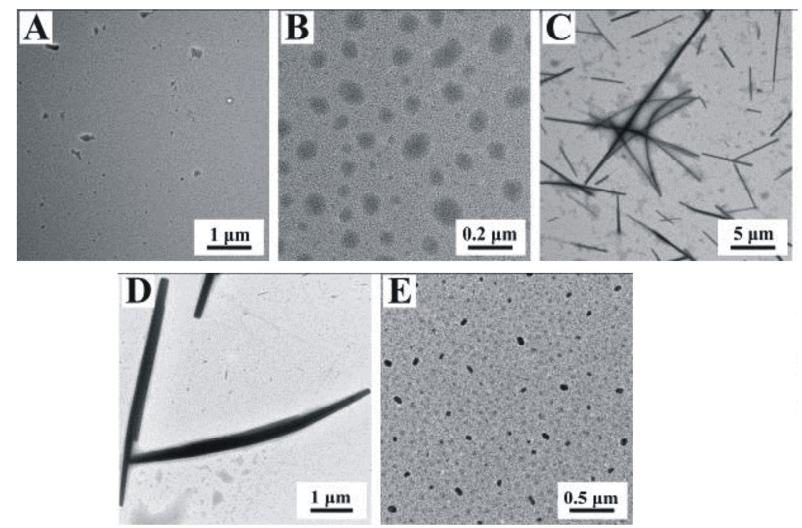

图 $5 \mathrm{PEG}_{45}-b-\mathrm{PGlu}_{24}$ 与 $\mathrm{Me}[4] \mathrm{H}$ 的不同物质的量比下在水中 电荷复合自组装的 TEM 照片 $(\mathrm{pH}=6.0)$

Figure 5 TEM images of the self-assembly of $\mathrm{PEG}_{45}-b$ - $\mathrm{PGlu}_{24}$ charge-conjugated $\mathrm{Me}[4] \mathrm{H}$ with different molar ratios in water $(\mathrm{pH}=6.0)$

(A) $1: 0.1 ;$ (B) $1: 0.2 ;$ (C) $1: 0.5 ;$ (D) $1: 0.7 ;$ (E) $1: 1.0$

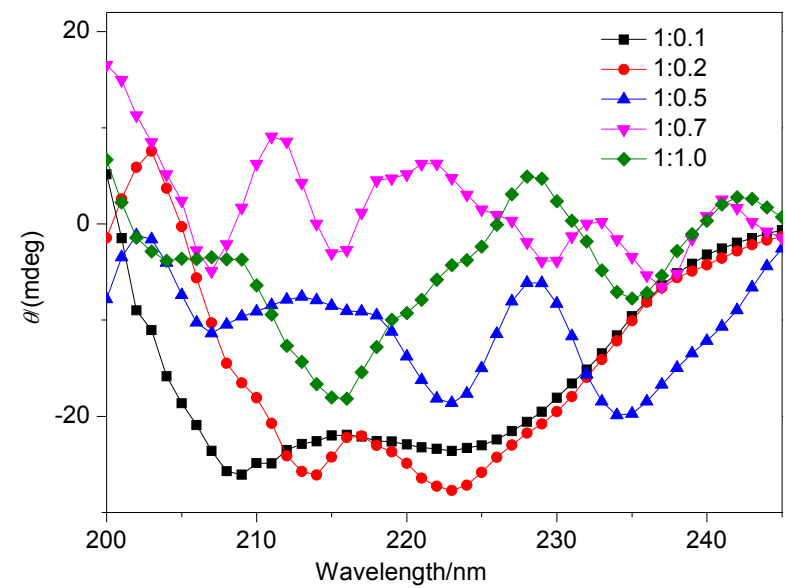

图 $6 \mathrm{PEG}_{45}-b-\mathrm{PGlu}_{24}$ 与 $\mathrm{Me}[4] \mathrm{H}$ 的不同物质的量比下在水中 电荷复合自组装的圆二色谱图 $(\mathrm{pH}=6.0)$

Figure $6 \mathrm{CD}$ spectra of self-assembly of $\mathrm{PEG}_{45}-b-\mathrm{PGlu}_{24}$ charge-conjugated $\mathrm{Me}[4] \mathrm{H}$ with different molar ratios in water $(\mathrm{pH}=6.0)$
结合表4的 $\zeta$-电位值结果和图6的圆二色谱结果，可 知在羧基与鎓离子之比分别为 $1: 0.1 、 1: 0.2$ 时, PGlu嵌 段依然有部分 $\alpha$-螺旋构象, 而此时加入的 $\mathrm{Me}[4] \mathrm{H}$ 并未 形成有效的 $\pi$ - $\pi$ 堆叠. 这可能是因为 $\mathrm{PGlu}_{24}$ 的螺旋保持能 力比 $\mathrm{PGlu}_{10}$ 更好, 这使得螺旋导向能力更强, 少量 $\mathrm{Me}[4] \mathrm{H}$ 无法通过 $\pi-\pi$ 堆叠作用形成平面结构; 加之在 $\mathrm{pH}=6.0$ 时, PEG- $b$-PGlu也相对亲水, 因此即使加入摩 尔分数为 0.2 的 $\mathrm{Me}[4] \mathrm{H}$, 也只能得到油滴状组装体. 当 $\mathrm{Me}[4] \mathrm{H}$ 物质的量比继续增加到 0.5 时, $\pi-\pi$ 堆叠才开始起 作用, 此时所得结果与 $\mathrm{PEG}_{45}-b-\mathrm{PGlu}_{10}$ 体系中 $\mathrm{Me}[4] \mathrm{H}$ 物 质的量比为 0.2 的结果类似. 但是, 由于亲水的PEG链段 相对较短, 因此在进一步增加 $\mathrm{Me}[4] \mathrm{H}$ 量到 1.0 后, 只能 得到较小的不规则胶束, 无法得到大的组装体.

表 $4 \mathrm{PEG}_{45}-b-\mathrm{PGlu}_{24}$ 与 $\mathrm{Me}[4] \mathrm{H}$ 的不同物质的量比下在水中 电荷复合自组装的 $\zeta$-电位值 $(\mathrm{pH}=6.0)$

Table $4 \zeta$-Potential values of self-assembly of $\mathrm{PEG}_{45}-b$-PGlu 24 charge-conjugated $\mathrm{Me}[4] \mathrm{H}$ with different molar ratios in water $(\mathrm{pH}=6.0)$

\begin{tabular}{cc}
\hline Glu $: \mathrm{Me}[4] \mathrm{H}$ & $\zeta$-Potential $/ \mathrm{mV}$ \\
\hline $1: 0.1$ & -19.10 \\
$1: 0.2$ & -15.13 \\
$1: 0.5$ & -7.19 \\
$1: 0.7$ & -6.83 \\
$1: 1.0$ & -4.42 \\
\hline
\end{tabular}

\section{4 溶液 $\mathrm{pH}$ 值对组装体形貌的影响}

选择 $\mathrm{PEG}_{45}-b-\mathrm{PGlu}_{24}$, 使其与 $\mathrm{Me}[4] \mathrm{H}$ 在水中进行电 荷复合自组装, $\mathrm{PEG}_{45}-b-\mathrm{PGlu}_{24}$ 与 $\mathrm{Me}[4] \mathrm{H}$ 的羒基与鎓离 子之比为 $1: 0.5$, 改变溶液的 $\mathrm{pH}$ 值(分别为 $3.0 、 4.0$ 、 $5.0 、 6.0 、 7.5 、 8.5)$, 研究 $\mathrm{pH}$ 值变化对组装体形貌的影 响. 当 $\mathrm{pH}$ 值为 3.0 时，得到的是 $10 \sim 20 \mathrm{~nm}$ 左右的小胶 束(图 7A), 表面只带很少的负电荷(表 5). 这是由于谷氨 酸的等电点为 3.2 , 在 $\mathrm{pH}$ 为 3.0 时, PGlu 电离很少, 无法 与 $\mathrm{Me}[4] \mathrm{H}$ 形成有效的电荷复合, 因此观察到的小胶束 是 $\mathrm{PEG}_{45}-b-\mathrm{PGlu}_{24}$ 或 $\mathrm{Me}[4] \mathrm{H}$ 自己组装而成的. 当 $\mathrm{pH}$ 值 达到 4.0 时, PGlu 带有的负电荷增加, 与少量螺烯形成 电荷复合, 但此时 PGlu的构象仍是螺旋结构为主, 而且 负电荷量较少，电荷中和作用较弱，使得 $\mathrm{Me}[4] \mathrm{H}$ 的 $\pi-\pi$ 堆叠并不显著, 因此形成长度 $1 \mu \mathrm{m}$ 左右的指针状组装 体(图 7B). 随着 $\mathrm{pH}$ 值增大, PGlu 链段的电离程度增加, 其刚性和疏水性减弱, 有利于电荷复合和 $\mathrm{Me}[4] \mathrm{H}$ 的 $\pi-\pi$ 堆叠, 因此指针状组装体的尺寸也随之增大(图 7C 7D). 当 $\mathrm{pH}$ 值达到 7.5 时, PGlu 链段更加柔软, 亲水性也 更好, 层状组装结构可以弯曲, 此时得到的是长度 $4 \sim 5$ $\mu \mathrm{m}$, 直径 $0.6 \sim 0.7 \mu \mathrm{m}$ 左右的纳米管(图 7E). 根据 $\zeta$-电 位结果(表 5), 推测纳米管的内外壁表面为 PEG 链段亲 水层, 疏水内管壁的表层则是 PGlu, 管壁内层由 
$\mathrm{Me}[4] \mathrm{H} \pi-\pi$ 堆叠而成. 当 $\mathrm{pH}$ 值达到 8.5 时, PGlu 嵌段电 离程度高(表 5), 几乎完全亲水, 因此, 与 $\mathrm{Me}[4] \mathrm{H}$ 电荷 复合后只能形成直径约为 $0.3 \mu \mathrm{m}$ 左右的油滴状形貌(图 $7 F)$.

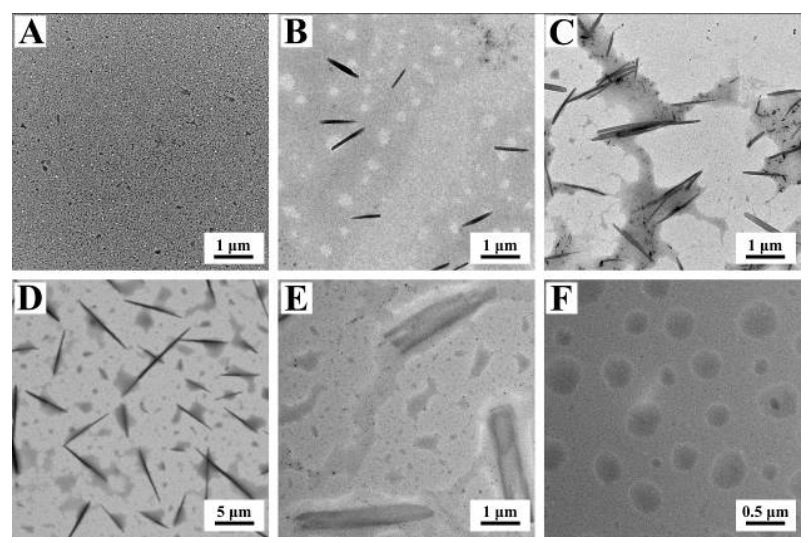

图 $7 \mathrm{PEG}_{45}-b-\mathrm{PGlu}_{24}$ 与 $\mathrm{Me}[4] \mathrm{H}$ 在 $\mathrm{pH}$ 值不同的水中电荷复合 自组装的 TEM 照片(羧基与鎓离子之比为 $1: 0.5$ )

Figure 7 TEM images of the self-assembly of $\mathrm{PEG}_{45}-b$ - $\mathrm{PGlu}_{24}$ charge-conjugated $\mathrm{Me}[4] \mathrm{H}$ in water of different $\mathrm{pH}$ values (carboxyl group and onium ion ratio is $1: 0.5)$

(A) $\mathrm{pH}=3.0$; (B) $\mathrm{pH}=4.0$; (C) $\mathrm{pH}=5.0$; (D) $\mathrm{pH}=6.0$; (E) $\mathrm{pH}=7.5$; (F) $\mathrm{pH}=8.5$

表 $5 \mathrm{PEG}_{45}-b-\mathrm{PGlu}_{24}$ 与 $\mathrm{Me}[4] \mathrm{H}$ 在不同 $\mathrm{pH}$ 值的水中电荷复合 自组装的 $\zeta$-电位值(羒基与鎓离子之比为 $1: 0.5$ )

Table $5 \zeta$-Potential values of self-assembly of $\mathrm{PEG}_{45}-b$-PGlu 24 charge-conjugated $\mathrm{Me}[4] \mathrm{H}$ in water of different $\mathrm{pH}$ values (carboxyl group and onium ion ratio is $1: 0.5$ )

\begin{tabular}{cc}
\hline $\mathrm{pH}$ & $\zeta$-Potential $/ \mathrm{mV}$ \\
\hline 3.0 & -1.46 \\
4.0 & -4.34 \\
5.0 & -6.48 \\
6.0 & -7.19 \\
7.5 & -10.03 \\
8.5 & -15.93 \\
\hline
\end{tabular}

\section{2 结论}

与传统的两亲嵌段共聚物组装体形貌随亲疏水链 段体积分数变化的规律不同, PEG- $b-\mathrm{PGlu}$ 与 $\mathrm{Me}[4] \mathrm{H}$ 电 荷复合物的形貌受螺烯 $\pi-\pi$ 堆叠作用、PGlu 电荷强度、 PGlu 构象、疏水部分体积分数等因素的共同影响. 当 $\pi-\pi$ 堆叠作用占主导地位时才能得到以指针状和条带状 等平面结构为主的组装形貌. $\pi-\pi$ 堆叠越显著, 得到的指 针状和条带状组装体尺寸也越大, 直至最后形成大尺寸 的飘带状组装体. 本研究填补了电荷复合自组装领域 中, 刚硬构象的分子相互复合时形貌变化规律研究的空 白, 也对两亲聚肽嵌段聚合物通过电荷复合作用搭载刚 硬构象的功能分子进行药物递送、制备功能化组装体等 应用有很好的参考价值.

\section{3 实验部分}

\section{1 仪器与试剂}

$L$-谷氨酸购自吉尔生化(上海)有限公司, 生化试剂; 苯甲醇, 四氢呋喃(THF), 碳酸氢钠, 三氟乙酸和氢溴 酸冰醋酸溶液购自阿达玛斯试剂有限公司, 试剂纯度 均为分析纯; 浓硫酸和浓盐酸购自国药集团化学试剂 有限公司, 分析纯; 无水乙醇和无水乙醚购自上海泰 坦科技股份有限公司, 试剂纯度均为分析纯; $N, N$-二甲 基甲酰胺(DMF)购自 Sigma-Aldrich 上海贸易有限公司, 超干溶剂; 三光气购自上海多点化工有限公司, 化学 纯; 正已烷购自百灵威科技有限公司，超干溶剂; 金属 钠购自上海凌峰化学试剂有限公司, 化学纯; 二苯甲 酮购自上海麦克林生物科技有限公司，分析纯; 端氨 基聚乙二醇 $\left(\mathrm{mPEG}-\mathrm{NH}_{2}\right.$, 数均分子量 $M_{n}$ 为 $\left.2.00 \times 10^{3}\right)$, 购自厦门赛诺邦格生物科技有限公司. 实验中用到的 四氢呋喃经除水处理，置于手套箱备用. 其余试剂不 做处理, 直接使用.

PL-GPC-50 plus 型凝胶渗透色谱仪(GPC, 美国瓦 里安公司), 标样为 $\mathrm{PBLG}$, 溶剂为 DMF (含 $0.02 \mathrm{~mol} / \mathrm{L}$ 澳化锂), 测试温度为 $50{ }^{\circ} \mathrm{C}$, 聚合物质量浓度约为 2 $\mathrm{mg} / \mathrm{mL}$; Avance 400 型核磁共振氢谱仪 $\left({ }^{1} \mathrm{H}\right.$ NMR, 德国 布鲁克公司), 以氛代氯仿 $\left(\mathrm{CDCl}_{3}\right)$ 为溶剂, 以四甲基硅 烷(TMS)为内标物; JEM-1400型透射电子显微镜(TEM, 日本电子株式会社)；J-810 型圆二色谱仪(CD，日本佳 士科公司); Zetasizer NanoZS ZEN3600 型纳米粒度电 位仪 ( $\zeta$-Potential, 英国马尔文公司), 测试温度为 $25{ }^{\circ} \mathrm{C}$, 平衡时间 $2 \mathrm{~min}$, 样品室为 DTS1070, 测试次数 3 次, 每次测量运行次数为 20 .

\section{$3.2 \mathrm{Me}[4] \mathrm{H}$ 的合成}

4-氮杂[4]螺烯合成参见文献[38]. 将 $229 \mathrm{mg} 4$-氮杂 [4]螺烯 $(1 \mathrm{mmol})$ 充分溶解于 $5 \mathrm{~mL}$ 二氯甲烷溶液, 之后 再缓慢滴加 $1 \mathrm{~mL}$ 碘甲烷. 滴加完毕后, 混合物室温摚 拌 $16 \mathrm{~h}$, 之后真空除去溶剂与未反应的碘甲烷. 所得残 余物溶于 $5 \mathrm{~mL}$ 二氯甲烷, 加入 $50 \mathrm{~mL}$ 乙醚, 出现大量黄 色沉淀. 所得沉淀过滤，并用乙醚洗涤，干燥后得产物 4-甲基-4-氮杂[4]螺烯鎓碘化物 Me[4]H $355 \mathrm{mg}$, 为黄色 粉末, 产率 96\%. ${ }^{1} \mathrm{H}$ NMR (DMSO- $\left.d_{6}, 400 \mathrm{MHz}\right) \delta: 10.12$ (d, $J=10.1 \mathrm{~Hz}, 1 \mathrm{H}), 9.54$ (d, $J=10.1 \mathrm{~Hz}, 1 \mathrm{H}), 8.91$ (d, $J=$ $10.1 \mathrm{~Hz}, 1 \mathrm{H}), 8.83(\mathrm{~d}, J=8.8 \mathrm{~Hz}, 1 \mathrm{H}), 8.64(\mathrm{~d}, J=8.8 \mathrm{~Hz}$, $1 \mathrm{H}), 8.37(\mathrm{~d}, J=8.8 \mathrm{~Hz}, 1 \mathrm{H}), 8.34(\mathrm{~d}, J=8.3 \mathrm{~Hz}, 1 \mathrm{H}), 8.31$ (d, $J=8.3 \mathrm{~Hz}, 1 \mathrm{H}), 8.25(\mathrm{~d}, J=8.8 \mathrm{~Hz}, 1 \mathrm{H}), 7.91 \sim 7.93$ (m, $J=7.9 \mathrm{~Hz}, 2 \mathrm{H}), 4.79$ (s, $J=4.8 \mathrm{~Hz}, 3 \mathrm{H})$.

\subsection{PEG-b-PGlu 的合成}

首先制备 $\gamma$-苄基- $L$-谷氨酸, 再以三光气法制备 
BLG-NCA 单体 ${ }^{[39]}$, 产物于手套箱中用无水四氢呋喃和 无水正己烷进行多次重结晶, 直至得到纯净白色针状晶 体, 密封保存于手套箱的冰箱中待用. 然后在手套箱中 准确称取所需 BLG-NCA 与 $\mathrm{mPEG}-\mathrm{NH}_{2}$ 于 $20 \mathrm{~mL}$ 直型螺 口瓶中, 用 $3 \mathrm{~mL}$ 无水 $\mathrm{DMF}$ 溶解, 在手套箱中进行反应. 得到的嵌段聚合物 PEG- $b$-PBLG 用冰无水乙醚沉淀, 再 用冰醋酸和三氟乙酸进行脱保护后, 经过透析和冻干处 理，最终得到所需嵌段聚合物 PEG- $b$-PGlu.

\section{4 组装体的制备}

称取 PEG- $b$-PGlu 于 $5 \mathrm{~mL}$ 直型螺口瓶中, 以超纯水 为溶剂, 配制成 $1 \mathrm{mg} / \mathrm{mL}$ 的溶液, 按照不同的物质的量 比向溶液中加入 $\mathrm{Me}[4] \mathrm{H}$ 水溶液, 用盐酸调节溶液的 $\mathrm{pH}$ 值, 在摚拌状态下得到稳定的组装体溶液.

\section{辅助材料 (Supporting Information) PEG- $b$-PGlu 与} $\mathrm{Me}[4] \mathrm{H}$ 的 GPC、 ${ }^{1} \mathrm{H}$ NMR 数据. 这些材料可以免费从本 刊网站(http://sioc-journal.cn/)上下载.

\section{References}

[1] Gröhn, F. Soft Matter 2010, 6, 4296.

[2] Rybtchinski, B. ACS Nano 2011, 5, 6791.

[3] Faul, C. F. Acc. Chem. Res. 2014, 47, 3428.

[4] Kim, B. G.; Kim, M. S.; Kim, J. ACS Nano 2010, 4, 2160.

[5] Malinsky, J. E.; Jabbour, G. E.; Shaheen, S. E.; Anderson, J. D.; Richter, A. G.; Marks, T. J.; Armstrong, N. R.; Kippelen, B.; Dutta, P.; Peyghambarian, N. Adv. Mater. 1999, 11, 227.

[6] Palma, A.; Satta, M. J. Chem. Theory Comput. 2016, 12, 4042.

[7] Li, Y.; Zhang, X.; Cao, D. J. Phys. Chem. B 2013, 117, 6733.

[8] Zhao, Y.; Mei, L.; Lu, Q. Langmuir 2008, 24, 3937.

[9] Wicklein, A.; Ghosh, S.; Sommer, M.; Würthner, F.; Thelakkat, M. ACS Nano 2009, 3, 1107.

[10] Wang, C.; Guo, Y.; Wang, Z.; Zhang, X. Langmuir 2010, 26, 14509.

[11] Yao, Y.; Zhang, L.; Leydecker, T.; Samorì, P. J. Am. Chem. Soc. 2018, 140, 6984.

[12] Liu, W.; Liu, J.; Liu, W.; Li, T. J. Agric. Food Chem. 2013, 61, 4133.

[13] Omura, Y.; Kyung, K. H.; Shiratori, S.; Kim, S. H. Ind. Eng. Chem. Res. 2014, 53, 11727.

[14] Fang, R.; Zhang, H.; Yang, L.; Wang, H.; Tian, Y.; Zhang, X.; Jiang, L. J. Am. Chem. Soc. 2016, 138, 16372.

[15] Pappa, A. M.; Inal, S.; Roy, K.; Zhang, Y. ACS Appl. Mater. Inter. 2017, 9, 10427.

[16] Dochter, A.; Garnier T.; Pardieu, E.; Chau, N. T. T.; Maerten, C.; Senger, B.; Schaaf, P.; Jierry, L.; Boulmedais, F. Langmuir 2015, 31,10208 .
[17] Bianchi, R. C.; Silva, E. R. D.; Antonia, L. H. D.; Ferreira, F. F.; Alves, A. W. Langmuir 2014, 30, 11464.

[18] Deng, T.; Wang, J.; Li, Y. Y.; Han, Z. H.; Peng, Y. N.; Zhang, J.; Gao, Z.; Gu, Y. Q.; Deng, D. W. ACS Appl. Mater. Interfaces 2018, $10,27657$.

[19] Huang, X. H.; Jeong, Y. I.; Moon, B. K.; Zhang, L. D.; Kang, D. H.; Kim, I. Langmuir 2013, 29, 3223.

[20] Bui, L.; Abbou, S.; Ibarboure, E.; Guidolin, N.; Staedel, C.; Toulme, J. J.; Schatz, C. J. Am. Chem. Soc. 2012, 134, 20189.

[21] Kumar, R. J.; Macdonald, J. M.; Singh, T. B.; Waddington, L. J.; Holmes, A. B. J. Am. Chem. Soc. 2011, 133, 8564.

[22] Zhang, Y.; Yin, Q.; Lu, H.; Xia, H.; Lin, Y.; Cheng, J. ACS Macro Lett. 2013, 2, 809.

[23] Shaikh, A. Y.; Das, S.; Pati, D.; Dhaware, V.; Sen, G. S.; Hotha, S. Biomacromolecules 2014, 15, 3679.

[24] Ramasamy, T.; Choi, J. Y.; Cho, H. J.; Umadevi, S. K.; Shin, B. S.; Choi, H. G.; Yong, C. S.; Kin, J. O. Pharm. Res. 2015, 32, 1947.

[25] He, W.; Yan, J.; Jiang, W.; Li, S.; Qu, Y.; Niu, F.; Yan, Y.; Sui, F.; Wang, S.; Zhou, Y.; Jin, L.; Li, Y.; Ji, M.; Ma, P. X.; Liu, W.; Hou, P. Chem. Mater. 2018, 30, 7034.

[26] Yan, J.; Korolev, N.; Eom, K. D.; Tam, J. P.; Nordenskiöld, L. Biomacromolecules 2012, 13, 124

[27] Nishimura, T.; Yamada, A.; Umezaki, K.; Sawada, S. I.; Mukai, S. A.; Sasaki, Y.; Akiyoshi, K. Biomacromolecules 2017, 18, 3913.

[28] Ryu, K.; Lee, M.; Park, J.; Kim, T. ACS Appl. Bio. Mater. 2018, 1, 1496.

[29] Zhang, S.; Cai, C.-H.; Huang, Q.-J.; Lin, J.-P.; Xu, Z.-W. Acta Polym. Sin. 2018, 109 (in Chinese) (张朔，蔡春华，黄琦婧，林嘉平，徐占文，高分子学报，2018， 109.)

[30] Hu, Y.; Lin, R.; Zhang, P.; Fern, J.; Cheetham, A. G.; Patel, K.; Schulman, R.; Kan, C.; Cui, H. ACS Nano 2015, 10, 880.

[31] Schuster, N. J.; Hernández, S. R.; Bukharina, D.; Kotov, N. A.; Berova, N.; Nq, F.; Steiqerwald, M. L.; Nuckolls, C. J. Am. Chem. Soc. 2018, 140, 6235.

[32] Nishiyma, N.; Okazaki, S.; Cabral, H.; Miyamto, M.; Kato, Y.; Suqiyama, Y.; Nishio, K.; Matsumura, Y.; Kataoka, K. Cancer Res. 2003, 63, 8977.

[33] Lv, S.; Li, M.; Tang, Z.; Song, W.; Sun, H.; Liu, H.; Chen, X. Acto Biomater. 2013, 9, 9330.

[34] Song, W.; Tang, Z.; Li, M.; Lv, M.; Sun, H.; Deng, M.; Liu, H.; Chen, X. Acta Biomater. 2014, 10, 1392.

[35] Verbiest, T.; Elshocht, S. V.; Kauranen, M.; Hellemans, L.; Snauwaert, J.; Nuckolls, C.; Katz, T. J.; Persoons, A. Science 1999, $561,913$.

[36] Fang, L.; Lin, W.-B.; Shen, Y.; Chen, C.-F. Chin. J. Org. Chem. 2018, 38, 541 (in Chinese). (房蕾，林伟彬，沈望，陈传峰，有机化学, 2018, 38, 541.)

[37] Kaseyama, T.; Furumi, S.; Zhang, X.; Takeuchi, M. Angew. Chem., Int. Ed. 2011, 123, 3768.

[38] Hewlins, M, J, E.; Salter, R. Synthesis 2007, 2164.

[39] Yao, Y.; Li, W. W.; Wang, S. B.; Yan, D. Y.; Chen, X. S. Macromol. Rapid Commun. 2006, 27, 2019. 